\title{
Consenso de tromboprofilaxis en cirugía ginecológica
}

\section{Consensus on thromboprophylaxis in gynecological surgery}

\section{Grupo Desarrollador del Consenso de Tromboprofilaxis en Cirugía Ginecológica. Federación Colombiana de Obstetricia y Ginecología (FECOLSOG)*}

Recibido: 26 de febrero de 2021/Aceptado: 12 de marzo de 2021

\section{RESUMEN}

Objetivo: sintetizar la evidencia disponible en torno a las intervenciones para la prevención de eventos tromboembólicos venosos durante el cuidado perioperatorio de las mujeres con patología ginecológica benigna, buscando reducir la morbimortalidad asociada a esta condición.

Materiales y métodos: el grupo desarrollador estuvo integrado por profesionales de la salud, tomadores decisiones y un representante de las pacientes. Todos los participantes declararon sus conflictos de interés. Se construyeron preguntas clínicas contestables y se realizó la graduación de los desenlaces. Se elaboró la pesquisa de la información en Medline/PubMed, Embase, Epistemonikos y LILACS. La búsqueda también abarcó fuentes de literatura gris y se actualizó el 22 de septiembre de 2020 sin restricciones por fecha o idioma. Se implementó la aproximación GRADE (Grading of Recommendations Assessment, Development and Evaluation) para establecer la calidad de la evidencia y la fuerza de las recomendaciones, se realizó consenso formal acorde con la metodología RAND/UCLA. Previo a la publicación, el documento fue objeto de revisión por pares.

Correspondencia: Carlos Fernando Grillo-Ardila, Departamento de Obstetricia y Ginecología, Universidad Nacional de Colombia, Carrera 30 No. 45-03, Edificio 471, Oficina 205, Bogotá, Colombia. Tel.: +57 13165000 , ext. 15122 .

* Lista completa al final del documento.
Resultados: se presenta la versión corta del consenso "para la prevención de eventos tromboembólicos venosos durante el cuidado perioperatorio de las mujeres con patología ginecológica benigna”, junto a su evidencia de soporte y respectivas recomendaciones.

Conclusiones: como recomendaciones claves para la implementación, se proponen el uso de la escala de Caprini y el uso de intervenciones acorde con el nivel de riesgo perioperatorio de las pacientes.

Palabras clave: consenso, anticoagulantes, periodo perioperatorio, tromboembolia venosa, embolia pulmonar.

\section{ABSTRACT}

Objective: To draw a synthesis of the available evidence regarding interventions for the prevention of venous thromboembolic events during the perioperative care of women with benign gynecological pathology, with the aim of reducing the associated morbidity and mortality.

Materials and methods: The development group included healthcare professionals, decision-makers and one patient representative. All the participants stated their disclosures. Answerable clinical questions were built and outcomes were graded. The search for information was conducted in Medline, Embase, Epistemonikos and LILACS, but it also included grey literature and was updated on September 22, 2020, with no date or language restric- 
tions. The GRADE (Grading of Recommendations Assessment, Development and Evaluation) approach was used to determine the quality of the evidence and the strength of the recommendations. Formal consensus was developed in accordance with the RAND/UCLA methodology. The document was submitted to peer review before publication.

Results: The abbreviated version of the "consensus on the prevention of venous thromboembolic events during the perioperative care of women with benign gynecological pathology" is presented together with the supporting evidence and respective recommendations.

Conclusions: The key recommendations for implementation include the use of the Caprini scale and of interventions consistent with the individual perioperative risk level.

Key words: Consensus, anticoagulants, perioperative period, venous thromboembolism, pulmonary embolism.

\section{INTRODUCCIÓN}

La enfermedad tromboembólica venosa representa una de las principales complicaciones en el periodo perioperatorio en las mujeres con cirugía ginecológica (1). En una importante proporción (14\%), la enfermedad tromboembólica venosa se manifiesta como trombosis venosa profunda (TVP) o embolia pulmonar y, al igual que otros eventos cardiovasculares (v. g. infarto agudo de miocardio y ataque cerebrovascular isquémico), el tromboembolismo venoso (TEV) incrementa ostensiblemente la probabilidad de morir (1,2). Cerca del $10 \%$ de las pacientes con diagnóstico de TVP y el $35 \%$ de las mujeres con diagnóstico de tromboembolismo pulmonar fallecerán durante los primeros 90 días del evento (3).

Pero el TEV no solo representa un problema de salud en términos de su mortalidad; esta entidad también repercute negativamente sobre la calidad de vida y la sostenibilidad de los sistemas de salud $(3,4)$. Las pacientes con antecedente de TEV reportan menores puntajes en la escala específica de calidad de vida (VT-QoL por sus siglas en inglés) para los dominios función física, salud general y emocional (4), al tiempo que requieren un mayor consumo de recursos relacionados con la atención sanitaria (US\$ 62.838 durante los cinco primeros años del evento) (3). No cabe duda, la enfermedad tromboembólica representa un problema de salud relevante para las mujeres en el mundo (3).

Se desconoce la frecuencia exacta de esta condición en pacientes con cirugía ginecológica (3). No obstante, se estima que durante la estancia hospitalaria y los tres primeros meses luego del procedimiento, 1,3\% de las pacientes experimentarán un episodio de TVP y un 0,4\% desarrollarán una embolia pulmonar (5). Carga de la enfermedad sustancialmente mayor cuando se compara con la población general (8 vs. 330 pacientes por cada 100.000 personas) (3), con un riesgo de recurrencia superior al $20 \%$ durante los cinco primeros años (6).

Múltiples factores afectan la probabilidad de padecer un evento tromboembólico venoso a lo largo de la vida reproductiva de la mujer $(2,4)$. Junto al embarazo (Razón de Oportunidad $(\mathrm{OR})=4,24$; Intervalo de Confianza (IC) $95 \%$ : 1,30-13,84) y el consumo de anticonceptivos orales $(\mathrm{OR}=4,03$; IC $95 \%:$ 1,83-8,89), la hospitalización (OR = 5,07; IC $95 \%$ : 3,12-8,23), la patología oncológica $(\mathrm{OR}=14,64$; IC $95 \%: 7,73-27,73)$ y la cirugía ginecológica $(\mathrm{OR}=18,95$; IC $95 \%$ : 9,22-38,97), quizás representan los principales factores vinculados al desarrollo de esta noxa $(2,4)$. Dada la magnitud del problema, la carga de la enfermedad, y a la luz de los vacíos del conocimiento (uno de cada cuatro profesionales de la salud no gradúan de forma apropiada el riesgo de enfermedad tromboembólica venosa y tres de cada cuatro prescriben de forma inapropiada las intervenciones preventivas) (7), resulta perentorio que el especialista en Obstetricia y Ginecología conozca la evidencia disponible en torno a las diferentes escalas para graduar el riesgo en pacientes con cirugía ginecológica, al tiempo que le compete la responsabilidad de conocer los efectos deseables 
e indeseables de las intervenciones, todo ello con el ánimo de brindar la mejor atención en salud (2).

Este consenso sintetiza la evidencia disponible para la prevención de eventos tromboembólicos venosos durante el cuidado perioperatorio de las mujeres con patología ginecológica benigna. La versión completa del documento se encuentra disponible para la consulta de los interesados en la Federación Colombiana de Asociaciones de Obstetricia y Ginecología (FECOLSOG).

\section{MATERIALES Y MÉTODOS}

Este documento es un insumo para los profesionales involucrados en el cuidado perioperatorio de las mujeres con patología ginecológica benigna, e incluye también aquellos que generan políticas en salud. Este consenso sintetiza la evidencia disponible en torno a las intervenciones para la prevención de eventos tromboembólicos venosos durante el cuidado perioperatorio de las mujeres con patología ginecológica benigna, a fin de reducir la morbimortalidad asociada a esta condición.

Este documento no aborda el manejo de las pacientes en periodo de gestación o puerperio ni el cuidado perioperatorio de mujeres que requieran profilaxis para un evento tromboembólico arterial, el tratamiento de un evento tromboembólico agudo o crónico, o el manejo de una complicación derivada de un evento tromboembólico. El grupo desarrollador (GD) estuvo conformado por profesionales pertenecientes al área de enfermería, medicina general, anestesiología y cirugía ginecológica. El GD también contó con el acompañamiento de los tomadores de decisiones y de una representante de los pacientes. Los profesionales en epidemiología clínica que hicieron parte del proyecto diseñaron la búsqueda sistemática de la información en asocio con el grupo revisor Cochrane de la Universidad Nacional de Colombia.

Todos los expertos declararon sus conflictos de interés. El alcance y los objetivos de este consenso se definió con la participación de los actores interesados, con el objeto de garantizar la aplicabilidad de las recomendaciones (8). A su vez, la versión preliminar del documento se socializó a través de la página oficial de la FECOLSOG, en donde se habilitó un correo institucional para recibir aportes al contenido. Las comunicaciones recibidas fueron direccionadas al GD para evaluar su pertinencia (9).

Una vez se perfeccionó el documento de alcance y objetivos, se definió el listado de preguntas clínicas por contestar, realizando para ello un ejercicio no formal de priorización. Las preguntas fueron estructuradas en formato PICO (población, intervención, comparación y desenlaces) siguiendo los lineamientos de la guía metodológica (8) y acorde con el documento de alcances y objetivos (9). Cada desenlace fue graduado siguiendo la metodología GRADE (10). Las preguntas PICO fueron socializadas con los grupos de interés con el objeto de capturar sus aportes al proceso, así como para incluir la perspectiva de los pacientes (9).

Una vez surtidos los anteriores procesos, se dio paso a la búsqueda sistemática de la información, siguiendo los lineamientos propuestos por el grupo revisor Cochrane de la Universidad Nacional de Colombia, que realizó la pesquisa de la información en Medline; Medline In-Process \& Other NonIndexed Citations; Medline Daily Update; Embase; Epistemonikos y LILACS. La búsqueda electrónica se actualizó el 22 de septiembre de 2020, sin restricciones por fecha o tipo de idioma. La pesquisa también abarcó otras fuentes de conocimiento, como los archivos de la Revista Colombiana de Obstetricia y Ginecología, la revisión y lectura en "bola de nieve" de las referencias recuperadas e incluidas y, finalmente, por medio del contacto con expertos en la materia (11).

Se priorizó la inclusión de revisiones sistemáticas con metaanálisis y, de ser necesario, se procedió a buscar estudios primarios (9). Dos integrantes del GD realizaron independientemente la lectura de títulos, resúmenes y artículos en texto completo, con el ánimo de identificar aquellos estudios relevantes para responder las preguntas formuladas. Las discrepancias se resolvieron mediante consenso. Se 
utilizó la herramienta AMSTAR-2 (Ameasurement Tool to Assess Systematic Reviews) (12) como instrumento de evaluación crítica para las revisiones sistemáticas y cuando se trató de estudios primarios, los ensayos clínicos controlados fueron evaluados aplicando el instrumento de riesgo de sesgos versión 2.0 sugerido por el grupo Cochrane (13); finalmente, los estudios de exactitud diagnóstica fueron analizados utilizando el instrumento QUADAS-2 (Quality Assessment of Diagnostic Accuracy Studies) (14).

La síntesis de la evidencia se realizó utilizando el programa GRADEpro GDT app (Guideline Development Tool) (15) generando las respectivas tablas de evidencia. Finalmente, para la construcción de las recomendaciones se realizó un consenso formal según los lineamientos propuestos por la metodología RAND/UCLA (16), sopesando a lo largo de la reunión de trabajo y considerando para la redacción de las recomendaciones los beneficios, los efectos adversos, las preferencias de los pacientes y el potencial impacto de las intervenciones sobre los costos. Este documento fue revisado de forma independiente por dos pares evaluadores quienes comentaron las recomendaciones y la evidencia de soporte del consenso.

\section{RESULTADOS}

A continuación se presenta la síntesis de la evidencia recuperada, acompañada de su respectiva recomendación.

\section{Escala para graduar el riesgo de presentar un evento tromboembólico venoso en mujeres con cirugía ginecológica por patología benigna.}

\section{A. Índice de riesgo de Caprini}

Un estudio de cohorte prospectiva (17) evaluó el desempeño operativo del índice de Caprini en pacientes con cirugía mayor (tabla 1). Para ello, el estudio reclutó participantes mayores de 40 años, sin antecedente de oclusión de la vena cava inferior, requerimiento de anticoagulación plena, trombocitopenia, diátesis hemorrágica, infección activa e insuficiencia vascular periférica. Todos los participantes recibieron profilaxis perioperatoria con medias de compresión graduada y heparina no fraccionada por dos a cinco días luego del procedimiento. Evidencia de muy baja calidad sugiere que el índice de riesgo de Caprini quizás posee un buen desempeño (área bajo la curva 0,87; IC95\%: $0,81-0,93)$ a la hora de discriminar los pacientes que desarrollarán un evento tromboembólico venoso. Calidad de la evidencia muy baja: limitaciones en riesgo de sesgo y aplicabilidad de la evidencia.

\section{B. Índice de riesgo de Rogers}

Un estudio de cohorte retrospectiva (2) analizó los factores de riesgo asociados al desarrollo de un evento tromboembólico venoso en pacientes con cirugía mayor. Para ello se ensambló una cohorte de pacientes a quienes se les realizó un procedimiento quirúrgico mayor en el sistema gastrointestinal, tegumentario, músculo-esquelético o cardiovascular. El estudio excluyó participantes con cirugía del tracto urinario, sistema nervioso central, genital masculino o femenino, o bien, en los órganos de los sentidos. Evidencia de muy baja calidad sugiere que el índice de Rogers quizás posee un buen desempeño (índice C 0,76) a la hora de discriminar los pacientes que desarrollarán un evento tromboembólico venoso. Calidad de la evidencia muy baja: limitaciones en riesgo de sesgo y aplicabilidad de la evidencia.

\section{C. Índice American College of Chest Physicians (Colegio Americano de Médicos del Tórax)}

La búsqueda sistemática de la información no identificó estudios primarios o revisiones sistemáticas que evaluaran el desempeño operativo de este instrumento. 


\begin{tabular}{|c|c|c|c|}
\hline Recomendación 1 & \multicolumn{3}{|c|}{ Resumen } \\
\hline Condicional a favor & \multicolumn{3}{|c|}{$\begin{array}{l}\text { Se sugiere utilizar la escala de Caprini para clasificar el riesgo perioperatorio de } \\
\text { enfermedad tromboembólica venosa en pacientes con cirugía ginecológica por patología } \\
\text { benigna. } \\
\text { Calidad de la evidencia: muy baja } \oplus \bigcirc \bigcirc \bigcirc\end{array}$} \\
\hline Recomendación 2 & \multicolumn{3}{|c|}{ Resumen } \\
\hline Condicional en contra & \multicolumn{3}{|c|}{$\begin{array}{l}\text { No se sugiere utilizar la escala de Rogers para clasificar el riesgo perioperatorio de } \\
\text { enfermedad tromboembólica venosa en pacientes con cirugía ginecológica por patología } \\
\text { benigna. } \\
\text { Calidad de la evidencia: muy baja } \oplus \bigcirc \bigcirc \bigcirc\end{array}$} \\
\hline \multirow{6}{*}{$\begin{array}{l}\text { Punto de buena } \\
\text { práctica }\end{array}$} & \multicolumn{3}{|c|}{$\begin{array}{l}\text { En paciente con cirugía ginecológica por patología benigna, el riesgo de enfermedad } \\
\text { tromboembólica se puede clasificar de la siguiente manera (18): }\end{array}$} \\
\hline & $\begin{array}{l}\text { Categoría de riesgo } \\
\text { (Riesgo de TEV) }\end{array}$ & Puntaje de Caprini & Tipo de procedimiento \\
\hline & Muy bajo $(<1,5 \%)$ & 0 & Cirugía ambulatoria \\
\hline & Bajo $(1,5 \%)$ & $1-2$ & $\begin{array}{l}\text { Cirugía ambulatoria en } \\
\text { mujeres con factores } \\
\text { adicionales de riesgo de TEV }\end{array}$ \\
\hline & Moderado (3,0\%) & $3-4$ & $\begin{array}{l}\text { Cirugía por patología } \\
\text { benigna con internación }\end{array}$ \\
\hline & Alto $(6,0 \%)$ & $>5$ & Cirugía oncológica \\
\hline
\end{tabular}

\begin{tabular}{|c|c|c|c|}
\hline 1 Punto & 2 Puntos & 3 Puntos & 4 Puntos \\
\hline $\begin{array}{l}\text { - Edad entre 41-60 años } \\
\text { - Cirugía menor } \\
\text { - IMC > } 25 \mathrm{~kg} / \mathrm{m} 2 * \\
\text { - Edema en extremidades }\end{array}$ & $\begin{array}{l}\text { - Edad entre 61-74 años } \\
\text { - Cirugía mayor (abierta o } \\
\text { laparoscópica) > } 45 \text { min }\end{array}$ & $\begin{array}{l}\text { - Mayor de } 75 \text { años } \\
\text { - Historia de TEV† }\end{array}$ & $\begin{aligned} \text { - } & \mathrm{ACV} \ddagger<1 \text { mes } \\
\text { - } & \text { Fractura de cadera, } \\
& \text { pelvis y pierna }\end{aligned}$ \\
\hline $\begin{array}{l}\text { - Venas varicosas } \\
\text { - Embarazo o posparto } \\
\text { - Historia inexplicable o } \\
\text { recurrente de abortos } \\
\text { espontáneos }\end{array}$ & $\begin{array}{l}\text { - Malignidad } \\
\text { - Confinado en cama }>72 \\
\text { h }\end{array}$ & $\begin{array}{l}\text { - Historia familiar } \\
\text { de TEV } \\
\text { - Trombofilia }\end{array}$ & $\begin{array}{l}\text { - Lesión aguda de la } \\
\text { médula espinal }<1 \\
\text { mes }\end{array}$ \\
\hline $\begin{array}{l}\text { - Anticonceptivos orales o } \\
\text { reemplazo hormonal } \\
\text { - Sepsis }(<1 \text { mes }) \\
\text { - Enfermedad pulmonar grave, } \\
\text { que incluye neumonía }(<1 \\
\text { mes })\end{array}$ & $\begin{array}{l}\text { - Inmovilización con yeso } \\
\text { - Acceso venoso central }\end{array}$ & $\begin{array}{l}\text { - Trombocitopenia } \\
\text { inducida por } \\
\text { heparina }\end{array}$ & \\
\hline $\begin{array}{l}\text { - Función pulmonar anormal } \\
\text { - Infarto agudo de miocardio } \\
\text { - Insuficiencia cardiaca } \\
\text { congestiva ( }<1 \text { mes) } \\
\text { - Historia de enfermedad } \\
\text { inflamatoria intestinal }\end{array}$ & & & \\
\hline
\end{tabular}

* Índice de masa corporal, $\uparrow$ Tromboembolismo venoso, $\uparrow$ Accidente cerebrovascular. 


\section{Intervenciones no farmacológicas para} la prevención de eventos tromboembólicos venosos en mujeres con cirugía ginecológica por patología benigna

\section{A. Movimiento pasivo continuo}

\begin{tabular}{|c|c|}
\hline Recomendación 3 & Resumen \\
\hline $\begin{array}{c}\text { No se sugiere el movimiento } \\
\text { pasivo continuo para la } \\
\text { prevención de eventos } \\
\text { tromboembólicos venosos } \\
\text { en pacientes con cirugía } \\
\text { ginecológica por patología } \\
\text { benigna. }\end{array}$ \\
\hline Calidad de la evidencia: muy \\
baja $\oplus$ mo○
\end{tabular}

Una revisión sistemática (19) (AMSTAR 2: confianza moderada) evaluó la seguridad y la efectividad del movimiento pasivo continuo en pacientes con cirugía mayor. La intervención consistió en realizar movimiento pasivo continuo de las extremidades inferiores comenzando en el posoperatorio inmediato, durante los tres primeros días del procedimiento. Por su parte, el grupo control recibió terapia física convencional acompañada de deambulación precoz. Evidencia de muy baja calidad sugiere que, cuando se compara frente al grupo control, la prescripción de movimiento pasivo continuo quizás no reduce la incidencia de TVP (RR = 1,22; IC $95 \%$ : 0,841,79). Calidad de la evidencia muy baja: limitaciones en riesgo de sesgo, precisión y aplicabilidad de la evidencia.

\section{B. Medias de compresión graduada}

Una revisión sistemática (20) (AMSTAR 2: confianza alta) evaluó la seguridad y la efectividad del uso de medias de compresión graduada en pacientes con cirugía mayor. Los pacientes asignados a la intervención utilizaron las medias de compresión desde el ingreso a salas de cirugía, hasta la deambulación plena o por un periodo de 7 a 14 días. Evidencia de muy baja calidad sugiere que, cuando se compara frente a la no intervención, las medias de compresión graduada quizás reducen la incidencia de TVP (OR = 0,35; IC 95\%: 0,28-0,43) y tromboembolismo pulmonar $(\mathrm{OR}=0,38$; IC $95 \%$ : 0,15-0,96). Calidad de la evidencia muy baja: limitaciones en riesgo de sesgo, precisión y aplicabilidad de la evidencia.

\begin{tabular}{|c|c|}
\hline Recomendación 4 & Resumen \\
\hline Condicional a & $\begin{array}{c}\text { Se sugiere el uso de } \\
\text { medias de compresión } \\
\text { graduada en pacientes con } \\
\text { cirugía ginecológica por } \\
\text { patología benigna y riesgo } \\
\text { tromboembólico bajo. } \\
\text { Calidad de la evidencia: muy } \\
\text { baja } \oplus \bigcirc \bigcirc \bigcirc\end{array}$ \\
\hline Punto de buena & $\begin{array}{c}\text { Las medias de compresión } \\
\text { graduada se utilizan desde el } \\
\text { ingreso a salas de cirugía, hasta } \\
\text { la deambulación plena o por } \\
\text { un periodo de } 7 \text { días luego del } \\
\text { procedimiento, lo que permite } \\
\text { que la paciente se las retire por } \\
\text { segmentos cortos de tiempo, no } \\
\text { mayores a una hora. }\end{array}$ \\
\hline
\end{tabular}

\section{Medias de compresión graduada}

\section{hasta la rodilla versus hasta el muslo}

Una revisión sistemática (21) (AMSTAR 2: confianza moderada) comparó la efectividad del uso de medias de compresión graduada hasta la rodilla, frente al uso de medias que se extienden hasta el muslo, para la prevención de eventos tromboembólicos venosos. Tanto la intervención como el grupo control utilizaron las medias de compresión desde el ingreso al quirófano, hasta la deambulación plena o por un periodo de 5 días. Evidencia de muy baja calidad sugiere que quizás no existe una diferencia significativa entre los grupos, en términos de la frecuencia de TVP $(\mathrm{OR}=1,55$; IC $95 \%$ : 0,78-3,07). Calidad de la evidencia muy baja: limitaciones en riesgo de sesgo, precisión y aplicabilidad de la evidencia. 


\section{Compresión neumática intermitente}

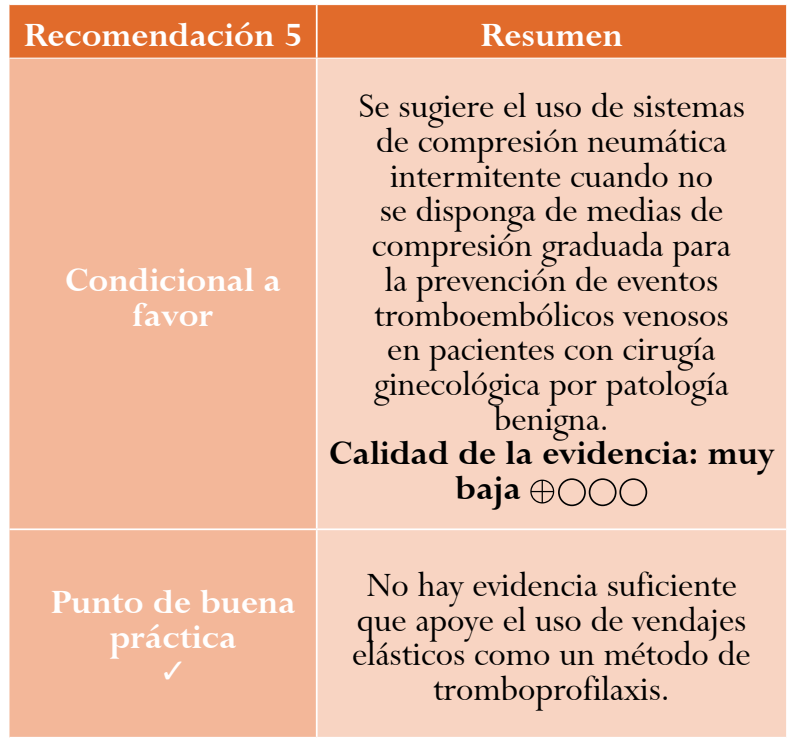

Una revisión sistemática (22) (AMSTAR 2: confianza críticamente baja) evaluó la seguridad y la efectividad del uso de la compresión neumática intermitente para el manejo de las pacientes con cirugía mayor ginecológica. La intervención de interés fue el uso de sistemas de compresión graduada, iniciando desde un día antes de la intervención, hasta los cinco primeros días luego del procedimiento.

a. Compresión neumática intermitente vs. grupo control. Evidencia de muy baja calidad sugiere que, cuando se compara frente al grupo control, el uso de compresión neumática intermitente quizás reduce la incidencia de TVP (RR = 0,33; IC 95\%: 0,16-0,66), sin que esto se acompañe aparentemente de una menor frecuencia de tromboembolismo pulmonar $(\mathrm{RR}=1,89$; IC $95 \%: 0,18-20,23)$.

b. Compresión neumática intermitente vs. tromboprofilaxis farmacológica. Evidencia de muy baja calidad sugiere que, cuando se compara frente a tromboprofilaxis farmacológica, la compresión neumática intermitente quizás no se asocia con una mayor incidencia de TVP (RR = 1,19; IC $95 \%$ : 0,42-3,44) o de tromboembolismo pulmonar $(\mathrm{RR}=7,47$; IC 95 \%: 0,42-132,78).

c. Compresión neumática intermitente más medias de compresión graduada vs. medias de compresión graduada. Evidencia de muy baja calidad sugiere que, cuando se compara frente al uso de medias de compresión graduada, la terapia combinada quizás no reduce la incidencia de TVP ( $R R=0,38$; IC $95 \%$ : 0,14-1,03) o de tromboembolismo pulmonar $(\mathrm{RR}=1,08$; IC $95 \%$ : 0,07-16,78). Calidad de la evidencia muy baja: limitaciones en riesgo de sesgo, precisión y aplicabilidad de la evidencia.

\section{E. Estimulación neuromuscular eléctrica}

\begin{tabular}{|c|c|}
\hline Recomendación 6 & Resumen \\
\hline Condicional en se sugiere el uso de la \\
contra \\
$\begin{array}{c}\text { Nostimulación neuromuscular } \\
\text { eléctrica para la prevención } \\
\text { de eventos tromboembólicos } \\
\text { venosos en pacientes con cirugía } \\
\text { ginecológica por patología } \\
\text { benigna. } \\
\text { Calidad de la evidencia: muy } \\
\text { baja } \oplus \bigcirc \bigcirc \bigcirc\end{array}$
\end{tabular}

Una revisión sistemática de la literatura (23) (AMSTAR 2: confianza moderada) evaluó la efectividad del uso de la estimulación neuromuscular eléctrica para el manejo de las pacientes con cirugía mayor. La intervención consistió en implementar el uso de un sistema de estimulación eléctrica neuromuscular dos horas antes del procedimiento, extendiéndose hasta el sexto día luego de la intervención.

a. Estimulación neuromuscular eléctrica vs. no intervención. Evidencia de muy baja calidad sugiere que, cuando se compara frente a no intervención, la estimulación neuromuscular eléctrica quizás se asocia con una menor frecuencia de TVP $(\mathrm{OR}=0,40$; IC $95 \%$ : 0,23-0,70), sin que esto se acompañe aparentemente de una menor frecuencia de tromboembolismo pulmonar (OR = 0,36; IC $95 \%$ : 0,12-1,07).

b. Estimulación neuromuscular eléctrica vs. medias de compresión graduada. Evidencia de muy baja calidad sugiere que, cuando se compara frente medias de compresión graduada, el uso de estimulación neuromuscular eléctrica quizás no se asocia con una menor incidencia de trombosis venosas profunda $(\mathrm{OR}=0,32$; IC $95 \%: 0,01-8,27)$ o de tromboembolismo pulmonar (OR = 0,32; IC $95 \%$ : 0,01-8,27). 
c. Estimulación neuromuscular eléctrica vs. heparina no fraccionada. Evidencia de muy baja calidad sugiere que, cuando se compara frente a la tromboprofilaxis farmacológica, la terapia de estimulación neuromuscular eléctrica quizás se asocia con una mayor incidencia de trombosis venosa profunda $(\mathrm{OR}=2,78 ;$ IC $95 \%$ : 1,19-6,48). La revisión no reportó el efecto de la intervención sobre la frecuencia de tromboembolismo pulmonar. Calidad de la evidencia muy baja: limitaciones en riesgo de sesgo, precisión y aplicabilidad de la evidencia.

\section{Intervenciones farmacológicas para la prevención de eventos tromboembólicos venosos en mujeres con cirugía ginecológica por patología benigna}

\section{A. Heparina (fraccionada o bajo peso molecular)}

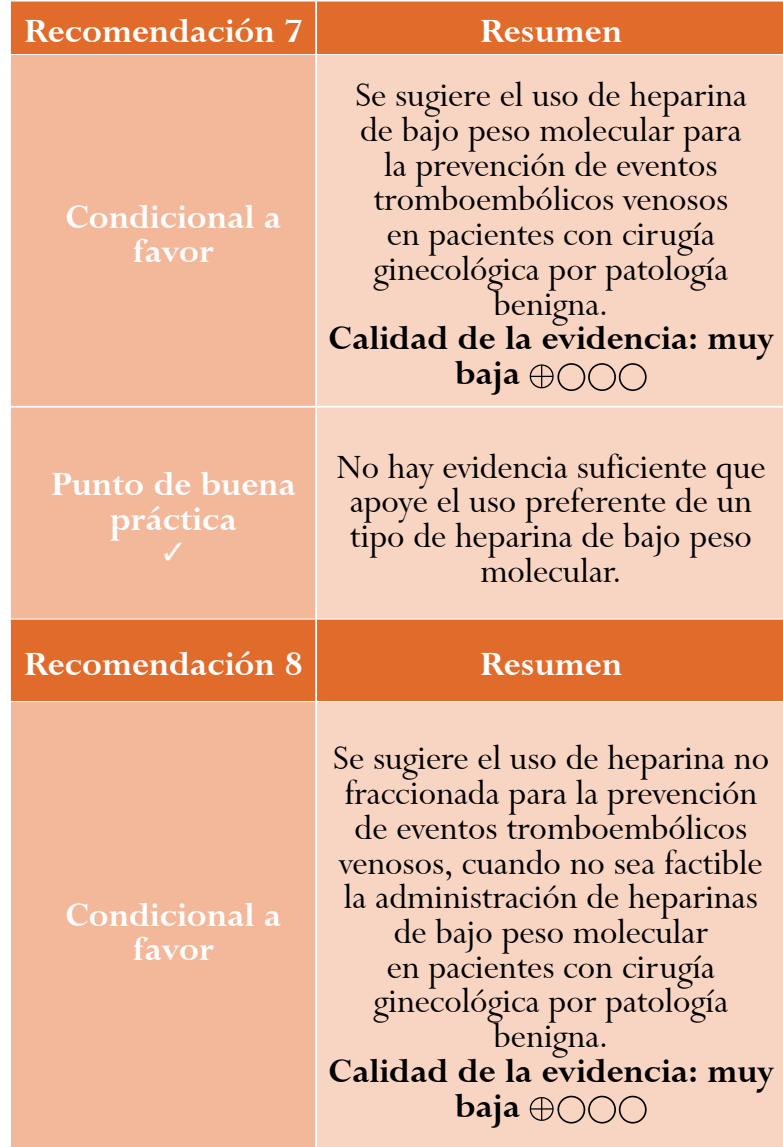

Una revisión sistemática de la literatura (24) (AMSTAR 2: confianza moderada) evaluó la seguridad y la efectividad del uso de heparina para el manejo de las pacientes con cirugía ginecológica mayor. La intervención consistió en el inicio de tromboprofilaxis farmacológica durante el perioperatorio inmediato (primeras 16 horas luego del procedimiento), hasta los 7 primeros días posteriores a la intervención.

a. Heparina vs. placebo. Evidencia de muy baja calidad sugiere que, cuando se compara frente al grupo control, la terapia con heparina quizás reduce la incidencia de TVP (OR =0,30; IC $95 \%$ : 0,12-0,77), pero no de tromboembolismo pulmonar $(\mathrm{OR}=2,13$; IC $95 \%$ : 0,42-10,91). No se encontraron diferencias aparentes entre los grupos, en la frecuencia de eventos adversos serios $(\mathrm{OR}=4,46$; IC $95 \%$ : 0,54-36,45).

b. Heparinas de bajo peso molecular vs. heparinas no fraccionadas. Evidencia de muy baja calidad sugiere que, cuando se compara frente al uso de heparina no fraccionada, la administración de heparina de bajo peso molecular quizás se asocia con una frecuencia similar de TVP $(\mathrm{OR}=1,03$; IC 95\%: 0,33-3,20) y de tromboembolismo pulmonar $(\mathrm{OR}=1,82$; IC $95 \%$ : 0,57-5,80). Tampoco se encontraron diferencias aparentes entre los grupos en la cantidad de sangrado durante el posoperatorio inmediato (DM = 6,75 mm; IC $95 \%: 65,33-78,83 \mathrm{~mm}$ ). Calidad de la evidencia muy baja: limitaciones en riesgo de sesgo, precisión y aplicabilidad de la evidencia.

\section{B. Heparina de bajo peso molecular comparado con heparina no fraccionada}

Una revisión sistemática (25) (AMSTAR 2: confianza moderada) comparó la seguridad y la efectividad de la heparina de bajo peso molecular frente a la administración de heparina no fraccionada, para el manejo de los pacientes con cirugía mayor. Evidencia de muy baja calidad sugiere que, cuando se compara frente a heparina no fraccionada, la heparina de bajo peso molecular quizás se asocia con 
una menor incidencia de trombocitopenia inducida por heparina $(\mathrm{RR}=0$,23; IC $95 \%$ : 0,07-0,73). Calidad de la evidencia muy baja: limitaciones en riesgo de sesgo, precisión y aplicabilidad de la evidencia.

\section{Acorde con el tipo de heparina de bajo peso molecular}

La búsqueda sistemática de la información no identificó ensayos clínicos controlados o revisiones sistemáticas para esta comparación.

\section{Tromboprofilaxis prequirúrgica comparada con inicio posquirúrgico}

\begin{tabular}{|c|c|}
\hline Recomendación 9 & Resumen \\
\hline $\begin{array}{l}\text { Condicional a } \\
\text { favor }\end{array}$ & $\begin{array}{c}\text { Se sugiere que la } \\
\text { tromboprofilaxis farmacológica } \\
\text { con heparina comience en } \\
\text { el posoperatorio inmediato } \\
\text { en pacientes con cirugía } \\
\text { ginecológica por patología } \\
\text { benigna. } \\
\text { Calidad de la evidencia: muy } \\
\text { baja } \oplus \bigcirc \bigcirc \bigcirc\end{array}$ \\
\hline $\begin{array}{l}\text { Punto de buena } \\
\text { práctica }\end{array}$ & $\begin{array}{l}\text { La heparina puede comenzar a } \\
\text { las } 12 \text { horas del posoperatorio. }\end{array}$ \\
\hline
\end{tabular}

Una revisión sistemática de la literatura (24) (AMSTAR 2: confianza moderada) comparó la seguridad y la efectividad de la administración preoperatoria de heparina (fraccionada o bajo peso molecular) frente a su inicio en el posoperatorio inmediato, para el manejo de las pacientes con cirugía ginecológica mayor. La intervención consistió en la administración de tromboprofilaxis farmacológica durante el preoperatorio inmediato (dos horas antes del procedimiento), en tanto que la comparación fue el inicio de esta terapia durante las primeras 16 horas posteriores a la cirugía. Evidencia de baja calidad sugiere que quizás no existen diferencias entre los grupos en términos de la incidencia de TVP (OR = 1,44; IC 95\%: 0,49$4,20)$, tromboembolismo pulmonar $(\mathrm{OR}=1,88$; IC $95 \%$ : 0,17-21,10) o en la frecuencia de hematoma en el sitio de punción $(\mathrm{OR}=0,30$; IC $95 \%$ : 0,061,51). Calidad de la evidencia muy baja: limitaciones en riesgo de sesgos y en la precisión.

\section{E. Tromboprofilaxis extendida con heparina}

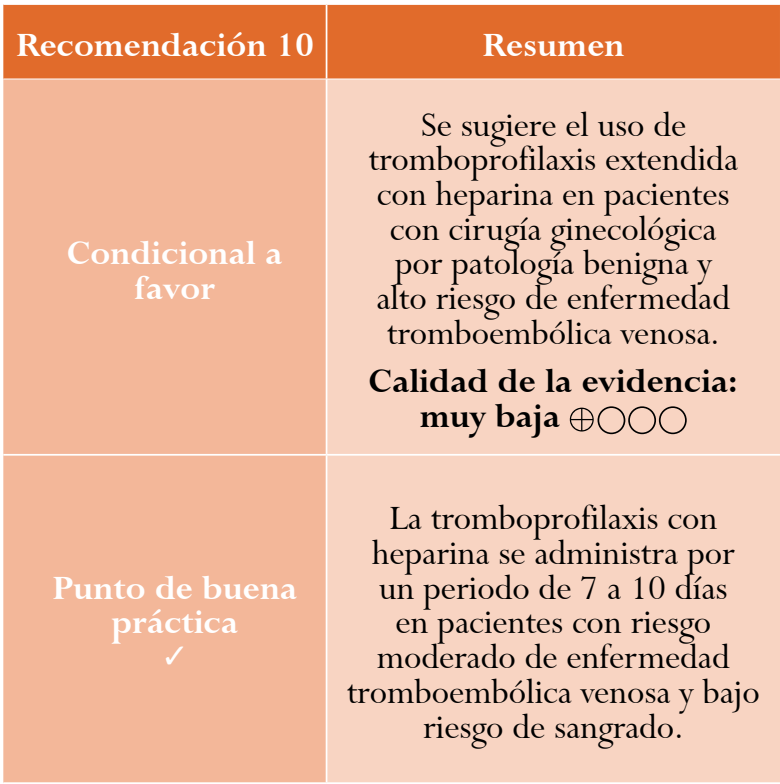

Una revisión sistemática (26) (AMSTAR 2: confianza moderada) evaluó la seguridad y la efectividad de la tromboprofilaxis extendida con heparina de bajo peso molecular en las pacientes con cirugía abdominal o pélvica. La intervención consistió en administrar heparina de bajo peso molecular durante el posoperatorio inmediato hasta por 28 días luego del procedimiento (grupo extendido), en tanto que el grupo control recibió tromboprofilaxis farmacológica exclusivamente durante la estancia hospitalaria (grupo no extendido). Evidencia de muy baja calidad sugiere que quizás la tromboprofilaxis extendida reduce la incidencia de TVP $(\mathrm{OR}=0,39$; IC $95 \%$ : 0,27-0,55). No se encontraron diferencias aparentes entre los grupos en la frecuencia de eventos adversos $(\mathrm{OR}=1,10$; IC 95\%: 0,67-1,81) o de muerte por enfermedad tromboembólica venosa $(\mathrm{OR}=1,15$ IC $95 \%$ : 0,72-1,84). Calidad de la evidencia muy baja: limitaciones en riesgo de sesgo, precisión y aplicabilidad de la evidencia. 
Una segunda revisión sistemática (27) (AMSTAR 2: confianza moderada) también evaluó la seguridad y la efectividad de la tromboprofilaxis extendida para el manejo de las pacientes con cirugía abdominal o pélvica. Evidencia de muy baja calidad sugiere que, cuando se compara frente a tratamiento habitual, la tromboprofilaxis extendida quizás no reduce la incidencia de tromboembolismo pulmonar $(R R=0,22 ;$ IC $95 \%$ 0,03-1,94). No se encontraron diferencias aparentes entre los grupos en la frecuencia de episodios de sangrado mayor $(\mathrm{RR}=0,63$; IC $95 \%$ : 0,17-2,28) o menor ( $R R=1,42$ : IC $95 \%$ : 0,68-2,99). Calidad de la evidencia muy baja: limitaciones en riesgo de sesgo, precisión y aplicabilidad de la evidencia.

\section{F. Fondaparina (pentasacárido)}

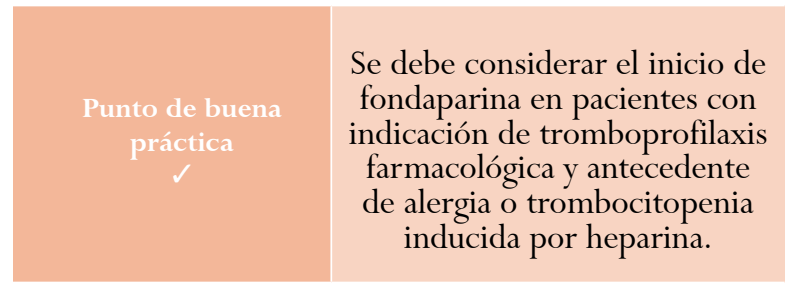

Una revisión sistemática de la literatura (6) (AMSTAR 2: confianza moderada) evaluó la seguridad y la efectividad del uso de fondaparina para el manejo de los pacientes con cirugía mayor. La intervención consistió en el inicio de fondaparina durante los 7 días previos a la intervención hasta 45 días luego del procedimiento quirúrgico.

a. Fondaparina vs. placebo. Evidencia de muy baja calidad sugiere que, cuando se compara frente al grupo control, la terapia con fondaparina quizás reduce la incidencia de tromboembolismo pulmonar $(\mathrm{RR}=0,16$; IC 95 \%: 0,04-0,62) y TVP $(\mathrm{RR}=0,25$; IC $95 \%$ : 0,15-0,40). Los pacientes asignados a fondaparina experimentaron una incidencia más alta de sangrado mayor $(\mathrm{RR}=2,56$; IC $95 \%$ : 1,48-4,44).

b. Fondaparina vs. tromboprofilaxis mecánica. Evidencia de muy baja calidad sugiere que, cuando se compara frente a la tromboprofilaxis mecánica, la terapia con fondaparina quizás no reduce la incidencia de cualquier evento tromboembólico venoso $(\mathrm{RR}=0,61 ;$ IC $95 \%$ : 0,22-1,67).

c. Fondaparina vs. heparina de bajo peso molecular. Evidencia de muy baja calidad sugiere que, cuando se compara frente al uso de heparinas de bajo peso molecular, la terapia con fondaparina quizás reduce la frecuencia de TVP $(\mathrm{RR}=0,54$; IC $95 \%$ : $0,40-0,71)$, sin que esto se vea reflejado en una menor incidencia de tromboembolismo pulmonar $(\mathrm{RR}=1,24$; IC $95 \%$ : 0,65-2,34). Los pacientes asignados a fondaparina experimentaron una incidencia más alta de sangrado mayor $(\mathrm{RR}=1,38$; IC $95 \%$ : 1,09-1,75). Calidad de la evidencia muy baja: limitaciones en riesgo de sesgo, precisión y aplicabilidad de la evidencia.

\section{G. Tromboprofilaxis farmacológica extendida más medias de compresión graduada}

Una revisión sistemática de la literatura (28) (AMSTAR 2: confianza críticamente baja) evaluó la seguridad y la efectividad del uso combinado de tromboprofilaxis farmacológica extendida acompañada de medias de compresión graduada para el manejo de las pacientes con cirugía abdominal o pélvica. La intervención consistió en administrar tromboprofilaxis farmacológica con heparina de bajo peso molecular, fondaparina o heparina no fraccionada acompañada de medias de compresión graduada (hasta la rodilla o el muslo), comenzando en el posoperatorio inmediato y extendiéndose (durante el posoperatorio) por un periodo de 21 a 42 días para la intervención farmacológica y hasta 7 días para el uso de las medias.

Evidencia de muy baja calidad sugiere que, cuando se compara frente a tromboprofilaxis farmacológica extendida, la terapia combinada quizás no reduce la incidencia de TVP sintomática $(\mathrm{RR}=1,51$; IC $95 \%: 0,16-14,32)$. Calidad de la evidencia muy baja: limitaciones en riesgo de sesgo, precisión y aplicabilidad de la evidencia. 


\section{H. Tromboprofilaxis farmacológica más compresión neumática intermitente}

Una revisión sistemática de la literatura (29) (AMSTAR 2: confianza moderada) evaluó la seguridad y la efectividad del uso combinado de tromboprofilaxis farmacológica en asocio con compresión neumática intermitente para el manejo de las pacientes con cirugía abdominal o pélvica. La intervención consistió en el inicio de compresión neumática intermitente dos horas antes del procedimiento, extendiéndose a lo largo de la estancia hospitalaria. Por su parte, la tromboprofilaxis farmacológica se inició durante el posoperatorio inmediato y se prolongó por 7 a 14 días luego de la intervención.

a. Tromboprofilaxis farmacológica en asocio con compresión neumática intermitente vs. compresión neumática intermitente. Evidencia de muy baja calidad sugiere que, cuando se compara con la compresión neumática intermitente, la terapia combinada quizás se asocia con una menor frecuencia de TVP $(\mathrm{OR}=0,52$; IC $95 \%$ : 0,33-0,82), a expensas de una mayor incidencia de sangrado (OR $=6,81$; IC $95 \%$ : 1,9923,28 para sangrado mayor y $\mathrm{OR}=5,04$; IC $95 \%$ : 2,36-10,77 para sangrado menor).

b. Tromboprofilaxis farmacológica más compresión neumática intermitente vs. tromboprofilaxis farmacológica. Evidencia de muy baja calidad sugiere que, cuando se compara con la tromboprofilaxis farmacológica, la terapia combinada quizás se asocia con una menor incidencia de tromboembolismo pulmonar $(\mathrm{OR}=0,39$; IC $95 \%: 0,23-0,64)$, sin diferencias aparentes entre los grupos, en la frecuencia de TVP $(\mathrm{OR}=1,02$; IC $95 \%$ : 0,29-3,54) o en los episodios de sangrado $(\mathrm{OR}=1,21$; IC 95\%: 0,35-4,18 para sangrado mayor y $\mathrm{OR}=0,80$; IC $95 \%$ : 0,30-2,14 para sangrado menor). Calidad de la evidencia muy baja: limitaciones en riesgo de sesgo, precisión y aplicabilidad de la evidencia.

\section{Diferentes estrategias de trombopro- filaxis para el manejo de las pacientes con cirugía ginecológica}

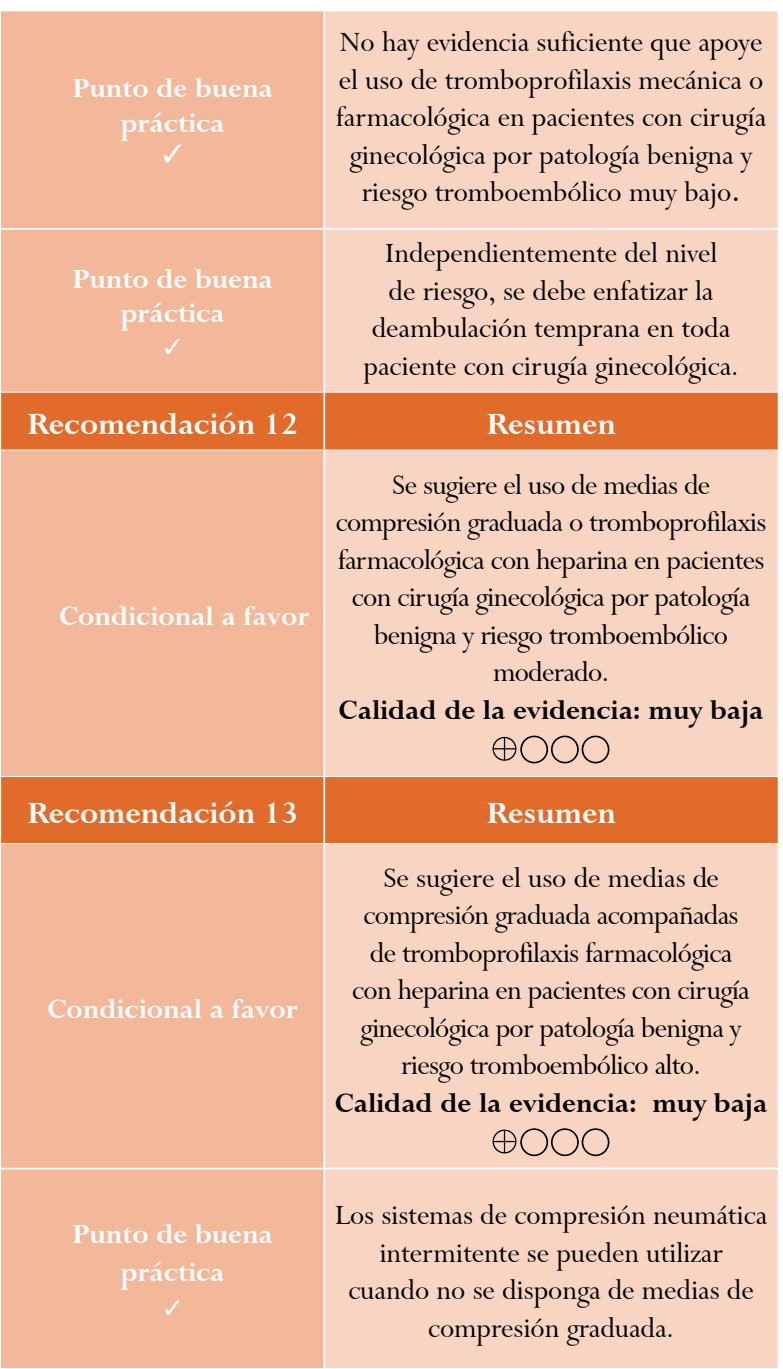

Un ensayo clínico controlado (30) (636 participantes) comparó la seguridad y la efectividad de tres estrategias de profilaxis para el manejo de las pacientes con cirugía ginecológica y riesgo alto o muy alto de enfermedad tromboembólica venosa. De acuerdo con la clasificación sugerida por el American College of Chest Physicians (Colegio Americano de Médicos del Tórax), las pacientes con tres factores se clasifican como alto riesgo, en tanto que aquellas con cuatro o más se consideran como con muy alto riesgo. Una vez verificados los criterios de inclusión y de exclusión, las participantes fueron asignadas a una de tres intervenciones: a) medias de 
compresión graduada más sistemas de compresión neumática, b) medias de compresión graduada más heparina de bajo peso molecular, y c) medias de compresión graduada, más sistemas de compresión neumática, más heparina de bajo peso molecular. Por su parte, el grupo control recibió medias de compresión graduada como monoterapia.

Las medias de compresión graduada (a la altura de la rodilla), se utilizaron desde el momento de la llegada de la paciente al quirófano hasta la deambulación plena. Por su parte, los sistemas de compresión neumática proporcionaron presión secuencial iniciando en el tobillo (45 $\mathrm{mm} / \mathrm{Hg})$, para luego desplazarse a hacia la pierna $(35 \mathrm{~mm} / \mathrm{Hg})$ y el muslo $(30 \mathrm{~mm} / \mathrm{Hg})$. Cada compresión duró 11 segundos y la duración de la relajación se ajustó espontáneamente de acuerdo con la velocidad de llenado venoso. La terapia comenzó 30 min antes de la cirugía y se interrumpió cuando la participante pudo deambular sin dificultad. En cuanto al grupo asignado para recibir heparinas de bajo peso molecular, esta se prescribió vía subcutánea periumbilical a dosis de tromboprofilaxis (dalteparina dosis de 5.000 Unidades Internacionales [UI] una vez al día), comenzando 12 horas después de la cirugía por un periodo no menor a 5 días.

a. Medias de presión graduada más sistemas de compresión neumática vs. medias de presión graduada. Evidencia de muy baja calidad sugiere que, cuando se compara frente al grupo control, la terapia combinada de medias más sistemas de compresión neumática quizás no reduce la incidencia global de tromboembolismo venoso $(\mathrm{OR}=0,51$; IC $95 \%$ : 0,24-1,10).

b. Medias de compresión graduada más heparina de bajo peso molecular vs. medias de compresión graduada. Evidencia de muy baja calidad sugiere que, cuando se compara frente al grupo control, la terapia combinada de medias más heparinas de bajo peso molecular quizás reduce la incidencia global de tromboembolismo venoso $(\mathrm{OR}=0,31$; IC $95 \%$ : 0,13-0,74).

c. Medias de compresión graduada, más sistemas de compresión más heparina de bajo peso molecular vs. medias de com- presión graduada. Evidencia de muy baja calidad sugiere que, cuando se compara frente al grupo control, la terapia combinada quizás reduce la incidencia global de tromboembolismo venoso (OR = 0,22; IC 95\%: 0,08$0,59)$. Calidad de la evidencia muy baja: limitaciones en riesgo de sesgo y en la precisión de los resultados.

\section{ACTUALIZACIÓN DEL CONSENSO}

Este consenso se deberá actualizar en los próximos tres años o antes, en caso de disponer de nueva evidencia que modifique de forma sustancial el contenido de las recomendaciones. Desde la perspectiva metodológica se sugiere:

1. Convocar un panel de expertos que lidere el proceso.

2. Priorizar aquellas preguntas que deberán ser actualizadas o desarrolladas.

3. Actualizar la pesquisa de la literatura.

4. Graduar la evidencia recuperada.

5. Actualizar o generar las nuevas recomendaciones a que haya lugar.

6. Reevaluar las recomendaciones claves para la implementación.

\section{CRITERIOS DE MONITORIZA- CIÓN Y AUDITORÍA}

1. Proporción de pacientes a las cuales se les aplicó la escala de Caprini para clasificar el riesgo perioperatorio de un evento tromboembólico venoso (>90\%).

2. Proporción de pacientes con cirugía ginecológica por patología benigna y riesgo tromboembólico bajo, a quienes se les prescribió el uso de medias de compresión graduada durante la estancia hospitalaria (>90\%).

3. Proporción de pacientes con cirugía ginecológica por patología benigna y riesgo tromboembólico moderado, a quienes se les prescribió el uso de medias de compresión graduada o tromboprofilaxis farmacológica durante la estancia hospitalaria (>90\%).

4. Proporción de pacientes con cirugía ginecológica por patología benigna y riesgo trom- 
boembólico alto, a quienes se les prescribió el uso de medias de compresión graduada y tromboprofilaxis farmacológica (>90\%).

\section{FINANCIACIÓN}

La elaboración del consenso contó con el apoyo de la Federación Colombiana de Obstetricia y Ginecología (FECOLSOG).

\section{AGRADECIMIENTOS}

Los autores agradecen a la doctora Ivonne Jeannette Díaz-Yamal, especialista en Ginecología y Obstetricia; especialista en Reproducción Humana, Unidad de Fertilidad Procreación Médicamente Asistida, Clínica de Marly, coordinadora de programa de Salud Reproductiva y Humana, Fundación Universitaria Sanitas, Bogotá (Colombia), y al doctor Jairo Amaya-Guío, médico cirujano; especialista en Obstetricia y Ginecología; especialista en Epidemiología Clínica. Profesor Titular, Facultad de Medicina, Universidad Nacional de Colombia, Bogotá (Colombia), por sus contribuciones al documento durante la revisión por pares.

\section{REFERENCIAS}

1. Cantrell LA, Garcia C, Maitland HS. Thrombosis and thromboprophylaxis in gynecology surgery. Clin Obstet Gynecol. 2018;61(2):269-77. https://doi.org/10.1097/ GRF.0000000000000355

2. Rogers SOJ, Kilaru RK, Hosokawa P, Henderson WG, Zinner MJ, Khuri SF. Multivariable predictors of postoperative venous thromboembolic events after general and vascular surgery: Results from the patient safety in surgery study. J Am Coll Surg. 2007;204(6):1211-21. https://doi.org/10.1016/j.jamcollsurg.2007.02.072

3. Heit JA, Spencer FA, White RH. The epidemiology of venous thromboembolism. J Thromb Thrombolysis. 2016;41(1):3-14. https://doi.org/10.1007/s11239-0151311-6

4. van Korlaar IM, Vossen CY, Rosendaal FR, Bovill EG, Cushman M, Naud S, et al. The impact of venous thrombosis on quality of life. Thromb Res. 2004;114(1):11-8. https://doi.org/10.1016/j.thromres.2004.04.007
5. Anderson DR, Morgano GP, Bennett C, Dentali F, Francis CW, Garcia DA, et al. American Society of Hematology 2019. Guidelines for management of venous thromboembolism: Prevention of venous thromboembolism in surgical hospitalized patients. Blood Adv. 2019;3(23):3898-944. https://doi.org/10.1182/ bloodadvances. 2019000975

6. Dong K, Song Y, Li X, Ding J, Gao Z, Lu D, et al. Pentasaccharides for the prevention of venous thromboembolism. Cochrane Database Syst Rev. 2016;10(10):CD005134. https://doi.org//10.1002/14651858.CD005134.pub3

7. Cabrera-Rayo A, Hernández-Díaz EJ, Guzmán-Rosales G, Laguna-Hernández G, Pliego-Reyes C, Zendejas-Villanueva JL, et al. Tromboprofilaxis en pacientes médicos y quirúrgicos: resultados de un estudio multicéntrico realizado en hospitales de la Ciudad de México. Med Int Méx. 2017;33(6):746-53. https://doi.org/10.24245/ mim.v33i6.1461

8. Ministerio de la Protección Social - Colombia, Centro de Estudios e Investigación en Salud de la Fundación Santa Fe de Bogotá. Guía metodológica para la elaboración de Guías de Práctica Clínica con Evaluación Económica en el Sistema General de Seguridad Social en Salud Colombiano; 2013. Disponible en: http://gpc. minsalud.gov.co/recursos/Documentos\%20compartidos/Guia_Metodologica_Web.pdf

9. Organización Panamericana de la Salud. Directriz para el fortalecimiento de los programas nacionales de guías informadas por la evidencia. Una herramienta para la adaptación e implementación de guías en las Américas. Washington, D.C.: OPS; 2018. Disponible en: http://iris.paho.org/xmlui/bitstream/ handle/123456789/49145/9789275320167_spa. pdf? sequence $=5$ \&isAllowed $=\mathrm{y}$;

10. Guyatt GH, Oxman AD, Kunz R, Atkins D, Brozek J, Vist G, et al. GRADE guidelines: 2. Framing the question and deciding on important outcomes. J Clin Epidemiol. 2011;64(4):395-400. https://doi.org/10.1016/j. jclinepi.2010.09.012

11. Higgins JPT, Green S, editor(s). Cochrane Handbook for Systematic Reviews of Interventions Version 5.1.0 (updated March 2011). Chichester (UK): John Wiley \& Sons, 2011. 
12. Shea BJ, Reeves BC, Wells G, Thuku M, Hamel C, Moran J, et al. AMSTAR 2: A critical appraisal tool for systematic reviews that include randomised or nonrandomised studies of healthcare interventions, or both. BMJ. 2017;358:j4008. https://doi.org/10.1136/ bmj.j4008

13. Higgins JPT, Savović J, Page MJ, Elbers RG, Sterne JAC. Assessing risk of bias in a randomized trial. In: Higgins JPT, Thomas J, Chandler J, Cumpston M, Li T, Page MJ WV, editors. Cochrane Handbook for Systematic Reviews of Interventions version 6.1 (updated September 2020). Cochrane, 2020. Disponible en: www.training.cochrane.org/handbook.

14. Whiting PF, Rutjes AWS, Westwood ME, Mallett S, Deeks JJ, Reitsma JB, et al. QUADAS-2: A revised tool for the quality assessment of diagnostic accuracy studies. Ann Intern Med. 2011;155(8):529-36. https:// doi.org/10.7326/0003-4819-155-8-20111018000009

15. GRADEpro GDT: GRADEpro Guideline Development Tool [Software]. McMaster University, 2015 (developed by Evidence Prime, Inc.). Disponible en: https://gradepro.org/

16. Vallejo-Ortega MT, Sánchez-Pedraza R, FelicianoAlfonso JE, García-Pérez MJ, Gutiérrez-Sepúlveda MP, Merchán-Chaverra RA. Manual Metodológico para la elaboración de protocolos clínicos en el Instituto Nacional de Cancerología. Bogotá: Instituto Nacional de Cancerología; 2016.

17. Bahl V, Hu HM, Henke PK, Wakefield TW, Campbell DAJ, Caprini JA. A validation study of a retrospective venous thromboembolism risk scoring method. Ann Surg. 2010;251(2):344-50. https://doi.org/10.1097/ SLA.0b013e3181b7fca6

18. Gould MK, Garcia DA, Wren SM, Karanicolas PJ, Arcelus JI, Heit JA, et al. Prevention of VTE in nonorthopedic surgical patients: Antithrombotic Therapy and Prevention of Thrombosis, 9th ed: American College of Chest Physicians Evidence-Based Clinical Practice Guidelines. Chest. 2012;141(2 Suppl):e227S-e277S. https://doi.org/10.1378/chest.11-2297

19. He ML, Xiao ZM, Lei M, Li TS, Wu H, Liao J. Continuous passive motion for preventing venous thromboembolism after total knee arthroplasty. Cochrane Database Syst Rev. 2014;(7):CD008207. https:// doi.org/10.1002/14651858.CD008207.pub3

20. Sachdeva A, Dalton M, Lees T. Graduated compression stockings for prevention of deep vein thrombosis. Cochrane Database Syst Rev. 2018; 11(11):CD001484. https://doi.org//10.1002/14651858.CD001484.pub4

21. Sajid MS, Desai M, Morris RW, Hamilton G. Knee length versus thigh length graduated compression stockings for prevention of deep vein thrombosis in postoperative surgical patients. Cochrane Database Syst Rev. 2012; (5):CD007162. https://doi. org//10.1002/14651858.CD007162.pub2

22. Feng JP, Xiong YT, Fan ZQ, Yan LJ, Wang JY, Gu ZJ. Efficacy of intermittent pneumatic compression for venous thromboembolism prophylaxis in patients undergoing gynecologic surgery: A systematic review and meta-analysis. Oncotarget. 2017;8(12):20371-79. https://doi.org/10.1002/14651858.CD007162.pub2

23. Hajibandeh S, Hajibandeh S, Antoniou GA, Scurr JR, Torella F. Neuromuscular electrical stimulation for the prevention of venous thromboembolism. Cochrane Database Syst Rev. 2017;11(11):CD011764. https:// doi.org//10.1002/14651858.CD011764.pub2

24. Oates-Whitehead RM, D'Angelo A, Mol B. Anticoagulant and aspirin prophylaxis for preventing thromboembolism after major gynaecological surgery. Cochrane database Syst Rev. 2003;(4):CD003679.

25. Junqueira DR, Zorzela LM, Perini E. Unfractionated heparin versus low molecular weight heparins for avoiding heparin-induced thrombocytopenia in postoperative patients. Cochrane Database Syst Rev. 2017;4(4):CD007557. https://doi. org/10.1002/14651858.CD007557.pub3

26. Felder S, Rasmussen MS, King R, Sklow B, Kwaan M, Madoff R, et al. Prolonged thromboprophylaxis with low molecular weight heparin for abdominal or pelvic surgery. Cochrane database Syst Rev. 2019;8:CD004318. https://doi.org/10.1002/14651858.CD004318.pub5

27. Rausa E, Kelly ME, Asti E, Aiolfi A, Bonitta G, Winter DC, et al. Extended versus conventional thromboprophylaxis after major abdominal and pelvic surgery: Systematic review and meta-analysis of randomized 
clinical trials. Surgery. 2018;164(6):1234-40. https:// doi.org/10.1016/j.surg.2018.05.028

28. Milinis K, Shalhoub J, Coupland AP, Salciccioli JD, Thapar A, Davies AH. The effectiveness of graduated compression stockings for prevention of venous thromboembolism in orthopedic and abdominal surgery patients requiring extended pharmacologic thromboprophylaxis. J Vasc Surg Venous Lymphat Disord. 2018;6(6):766-777.e2. https://doi. org/10.1016/j.jvsv.2018.05.020

29. Kakkos SK, Caprini JA, Geroulakos G, Nicolaides AN, Stansby G, Reddy DJ, et al. Combined intermittent pneumatic leg compression and pharmacological prophylaxis for prevention of venous thromboembolism. Cochrane database Syst Rev. 2016;9(9):CD005258. https://doi.org/10.1002/14651858.CD005258.pub3

30. Sang C-Q, Zhao N, Zhang J, Wang S-Z, Guo S-L, Li $\mathrm{S}-\mathrm{H}$, et al. Different combination strategies for prophylaxis of venous thromboembolism in patients: A prospective multicenter randomized controlled study. Sci Rep. 2018;8(1):8277. https://doi.org/10.1038/ s41598-018-25274-2

Declaración de independencia editorial. La entidad que auspició el consenso no influyó en el contenido de este documento. Los profesionales que formaron parte de esta iniciativa definieron de forma autónoma el contenido y la dirección de las recomendaciones.

\section{GRUPO DESARROLLADOR DEL CONSENSO}

Carlos Fernando Grillo-Ardila: médico cirujano; especialista en Obstetricia y Ginecología; magíster en Epidemiología Clínica. Profesor asistente, Departamento de Ginecología y Obstetricia, Universidad Nacional de Colombia; médico especialista, Grupo de Investigación Clínica y Epidemiológica del Cáncer, Instituto Nacional de Cancerología, Bogotá (Colombia). Coordinador académico del consenso.
Jimmy Castañeda Castañeda: médico cirujano; especialista en Ginecología y Obstetricia y Medicina Reproductiva, Hospital Militar Central, Unidad de Fertilidad P.M.A., Clínica de Marly, Bogotá (Colombia). Expresidente y director de Educación, Federación Colombiana de Obstetricia y Ginecología (FECOLSOG); profesor titular y coordinador Salud Sexual y Reproductiva, Universidad Militar Nueva Granada, Bogotá (Colombia). Coordinador temático del consenso.

Carlos Hernán Calderón-Franco: médico cirujano; especialista en Epidemiología; magíster en Epidemiología Clínica, Clínica Medilaser, Florencia (Colombia). Coordinador grupo metodológico del consenso.

Carolina María Muñoz-Sanjuán: médica cirujana, Pontificia Universidad Javeriana; MSc Medicina Alternativa. Directora Médica Simedics IPS Centro de Investigación Clínica, Bogotá (Colombia). Grupo metodológico del consenso.

\section{SOCIEDADES QUE PARTICIPA- RON EN EL CONSENSO}

\section{Federación Colombiana de Obstetricia y Ginecología (FECOLSOG)}

Juan Diego Villegas Echeverri: médico cirujano; especialista en Obstetricia y Ginecología, Universidad del Rosario; Fellow de Dolor pélvico crónico, Paul Perry Pelvic Pain Center - Brookwood Medical Center, Birmingham EE. UU.); especialista en Cirugía Ginecológica Laparoscópica, Universidad CES, Medellín (Colombia). Director científico, Unidad de Laparoscopia Ginecológica Avanzada y Dolor Pélvico (ALGIA), Pereira (Colombia). Experto clínico del consenso.

Orlando Flórez Victoria: médico cirujano; especialista en Ginecología y Obstetricia; especialista en Cirugía Endoscópica Ginecológica. Profesor aso- 
ciado, Departamento de Ginecología y Obstetricia, Facultad de Ciencias de la Salud, Universidad del Cauca. Popayán (Colombia). Experto clínico del consenso.

Álvaro Escobar: médico cirujano; especialista en Ginecología y Obstetricia, Universidad del Valle; especialista en Cirugía Ginecológica Mínimamente Invasiva, CES. Jefe del Servicio de Ginecología, Fundación Valle del Lili, Cali (Colombia); coordinador del Comité Cirugía Mínimamente Invasiva FECOLSOG, Valle del Cauca (Colombia). Experto clínico del consenso.

\section{Sociedad Colombiana de Anestesiología y Reanimación (S.C.A.R.E)}

Mauricio Vasco Ramírez: médico cirujano; especialista en Anestesiología, Cuidados Intensivos y Reanimación, Universidad Pontificia Bolivariana, Medellín (Colombia); estancia formativa en Anestesia Obstétrica en el Hospital CLINIC - Universidad de Barcelona (España); Scholarship en Simulación Médica, Center of Medical Simulation "STRATUS", Centro adscrito al Brigham and Women's Hospital, Harvard Medical School, Boston (EE. UU.); director de simulación clínica, Universidad CES, Medellín; presidente S.C.A.R.E., Medellín (Colombia). Experto clínico del consenso.

\section{Secretaría Distrital de Salud}

Amparo Leonor Ramírez Corredor: médico cirujano; especialista en Ginecología y Obstetricia; especialista en Bioética. Docente, Facultad de Medicina, Universidad El Bosque. Referente técnico Ginecología y Obstetricia, Subred Integrada de Servicios de Salud Sur Occidente E.S.E., Bogotá (Colombia). Experto clínico del consenso.
Jorge Eduardo Caro Caro: médico cirujano; especialista en Ginecología y Obstetricia; especialista en Auditoría y Calidad. Jefe de Ginecología y Obstetricia, Subred Integrada de Servicios de Salud Centro Oriente; profesor, Facultad de Medicina, Universidad Antonio Nariño, Bogotá (Colombia). Experto clínico del consenso.

Néstor Giraldo: médico cirujano, especialista en Ginecología y Obstetricia. Jefe Ginecología y Obstetricia Subred Integrada de Servicios de Salud Norte, Bogotá (Colombia). Experto clínico del consenso.

\section{Representante de los pacientes}

Paola Andrea Leal López: arquitecta; magíster en Medio Ambiente y Desarrollo; magíster en Administración de Empresas, Bogotá (Colombia). Representante de los pacientes en el consenso.

\section{Otros asistentes del panel}

\section{Representante de médicos generales}

Ricardo González: médico cirujano, Universidad de Ciencias Aplicadas y Ambientales U.D.C.A. Hospital Universitario de San Rafael de Tunja (Colombia). Experto clínico del consenso.

\section{Representante de Enfermería}

Johanna Duarte Romero: enfermera profesional, Universidad del Bosque; Hospital Militar Central, Bogotá (Colombia). Experta clínica del consenso.

\section{Semillero de Investigación}

Laura Natalia Naranjo Agudelo: estudiante de Medicina, Facultad de Medicina, Universidad Nacional de Colombia, Bogotá (Colombia). Apoyo Grupo Metodológico del consenso. 